\title{
ARTICLE
}

\section{Measurement of gamma rays under the high-energy p-Li neutron fields in RCNP}

\author{
So Kamada ${ }^{\mathrm{a}^{*}}$, Masashi Takada ${ }^{\mathrm{a}}$, Masayuki Hagiwara ${ }^{\mathrm{b}}$, Yosuke Iwamoto ${ }^{\mathrm{c}}$, Tatsushi Shima ${ }^{\mathrm{d}}$, Atsushi Tamii ${ }^{\mathrm{d}}$ \\ and Kichiji Hatanaka ${ }^{\mathrm{d}}$ \\ ${ }^{a}$ National Institute of Radiological Sciences, 4-9-1 Anagawa, Inage-ku, Chiba, 263-8555, Japan; ${ }^{b}$ High Energy Accelerator Research \\ Organization, 1-1 Oho Tsukuba, Ibaraki, 301-0801, Japan; ' Japan Atomic Energy Agency, 2-4 Shirakata, Shirane, Tokai, Ibaraki \\ 319-1195, Japan; ${ }^{d}$ Research Center for Nuclear Physics, Osaka University, 10-1 Mihogaoka, Ibaraki, Osaka, 567-0047, Japan
}

\begin{abstract}
We measured gamma-ray pulse-height spectrum of quasi-mono energetic neutron beam produced by the $\mathrm{p}-\mathrm{Li}$ reaction by using a $\mathrm{NaI}(\mathrm{Tl})$ scintillation detector, which has 7.62-cm diameter and 7.62-cm length, with time of flight (TOF) methods in Research Center for Nuclear Physics, Osaka, Japan. Prompt gamma-ray events in the TOF distribution are used to confirm our particle discrimination method. We can measure gamma ray events separately from neutrons in the neutron field.
\end{abstract}

\section{Keywords: gamma ray; neutron; time-of-flight method; cyclotron accelerator; NaI(Tl) scintillator}

\section{Introduction}

The quasi-monoenergetic neutron beam up to 400 $\mathrm{MeV}$ is now available in the Research Center for Nuclear Physics (RCNP) Osaka, Japan. The high-energy neutron source is utilized for the traditional nuclear physics, the study of radiation shielding, and the benchmark test of Monte Carlo transport calculation code. In addition, mono energetic neutron beam is widely used for several purposes, calibration of neutron dosimeters, biodosimetry, and study of the single event set up of semi-conductor devices. These neutron beams consist of not only neutrons but also gamma rays.

We may encounter the neutron and gamma rays external exposure in a variety of settings around the nuclear reactor, high-energy accelerator facilities, at nuclear accidents and at the altitude aviation. Gamma-ray backgrounds are accompanied with the neutron beam in the irradiation fields. The background consists of photons produced at neutron targets, and via accelerator components via inelastic scattering and capture reactions. Several neutron detectors and dosimeters can discriminate neutron events from photons; while, some detectors detect both photons and neutrons, such as ion chambers and passive dosimeters; (Passive detectors insensitive to gammas exist, e.g., superheated emulsions and track detectors, CR39). It is necessary to evaluate photon energy spectrum as well as main neutron spectrum. In the neutron fields, we measure photon energy spectrum using a $\mathrm{NaI}(\mathrm{Tl})$ scintillator with large detection efficiency and high energy resolution. We evaluated specification of particle discrimination of the $\mathrm{NaI}(\mathrm{Tl})$ scintillator to distinguish

*Corresponding author. Email: kamada-s@nmri.go.jp between photons and neutrons in the neutron fields. Organic liquid scintillators are usually used for having the particle discrimination of neutrons and gamma rays. Because the organic liquid scintillator is composed of hydrogen and carbon atoms with low atomic number, this scintillator shows a low intrinsic efficiency to gamma rays. In this study, $\mathrm{NaI}(\mathrm{Tl})$ scintillators whose pulse height spectra have total energy peaks due to the photoelectric effect are most useful for photon measurements. To evaluate characteristics of gamma rays in the high energy neutron field produced by the ${ }^{7} \mathrm{Li}(\mathrm{p}, \mathrm{n})$ reaction, we utilized the time-of-flight (TOF) method, and obtained the pulse height spectrum of prompt gamma rays with a $\mathrm{NaI}(\mathrm{Tl})$ scintillator by using the time-of-flight (TOF) method.

\section{Measurement}

\subsection{Detector}

We utilized a $\mathrm{NaI}(\mathrm{Tl})$ Scintillator, manufactured by Alpha Spectra, Inc., Arrowwest Court road, Grand Junction, Colorado, U.S.A., with 7.62-cm diameter and $7.62-\mathrm{cm}$ in length. Photons up to $175 \mathrm{MeV}$ can be measured with the $\mathrm{NaI}(\mathrm{Tl})$ scintillator, because electron range of $175 \mathrm{MeV}$ in a $\mathrm{NaI}$ crystal is same as the detector length, $7.5 \mathrm{~cm}[1]$. The detector was assembled with photomultiplier, R6559 manufactured by Hamamatsu Photonics Japan. The photomultiplier was surrounded by a magnetic shield. The detector bias applied to the photomultiplier was $-1900 \mathrm{~V}$. The $\mathrm{NaI}(\mathrm{Tl})$ scintillator has a good energy resolution $(\sim 7.5 \%$ for 662 $\mathrm{keV}$ gamma rays). The rise time of the anode pulse from photomultiplier is $\sim 50 \mathrm{~ns}$, so we can use the $\mathrm{NaI}(\mathrm{Tl})$ 
with time of flight measurement.

\subsection{High-energy neutron fields in RCNP}

Our experiments were performed at high-energy neutron fields produced by 137 and $200 \mathrm{MeV}$ protons transported to the $100 \mathrm{~m}$ tunnel next to the Neutron experimental hall from the ring cyclotron at RCNP. The beam frequencies were 11.6 MHz and 13.4 MHz for 137 $\mathrm{MeV}$ and $200 \mathrm{MeV}$ protons, respectively. Moreover, the beam chopper was available and the chopping ratio was $1 / 16$. The beam swinger is also available the target angles respect to the beam varies from $0 \mathrm{deg}$. to $30 \mathrm{deg}$. We utilized isotopic enriched lithium-7 (99.75\%) as a target. ${ }^{7} \mathrm{Li}(\mathrm{p}, \mathrm{xn})$ reaction create high-energy sharp peak at hundreds of $\mathrm{MeV}$. This neutron energy spectrum comprises of high-energy peak and continuum [2]. Proton beams through the lithium target were bent by the bending magnet and down to beam dump. We obtained incident proton beam fluxes from integration of proton beams into beam dump. The scintillation detector was set $15 \mathrm{~m}$ downstream of the lithium target. The signals from the photomultiplier were fed into the electronic circuits and then processed with the KODAQ system (Kakuken Online Data AcQisition system) [3] in the event-by-event mode. For each event, we collected the integrated charge of the anode signal measured by charge sensitive analogue to digital converter LeCroy 2249W (manufactured by LeCroy, Chestnut Ridge, NY, USA). On the other hand we recorded the time difference between the chopper signal from the cyclotron and the detector. In order to obtain the time-difference, we utilized the Time to Amplitude Converter (TAC), which produce a voltage pulse proportional to the time difference between its start input and its stop input. The TAC outputs were collected by the peak-sensitive analogue to digital converter ORTEC AD811 (manufactured by ORTEC, Oak Ridge, TN, U.S.A.) in list mode.

\subsection{Pulse height calibration}

The authors calibrated pulse height against electron energy, which is correlated with photo-peak energy using gamma-ray standard sources $\left({ }^{22} \mathrm{Na},{ }^{60} \mathrm{Co}\right)$ and natural background gamma-ray $\left({ }^{40} \mathrm{~K}(1.461 \mathrm{MeV})\right.$, $\left.{ }^{208} \mathrm{Tl}(2.615 \mathrm{MeV})\right)$ observed in measurement.

\subsection{Time of flight measurement}

Prompt gamma-ray events were selected from the events due to the neutrons using the two-dimensional plot pulse-height $v s$. TOF as shown in Figures 1(A), (B) and $(\mathbf{C})$ measured in the $\mathrm{p}$-Li neutron beam at the 0,10 and $25 \mathrm{deg}$. respect to the proton beam of $200 \mathrm{MeV}$. The horizontal and vertical axis indicates time difference and pulse height of signals from the photomultiplier, respectively. The time range per channel was $0.51 \mathrm{~ns}$. The peak around 1300 channel in TOF axis is a prompt gamma-ray peak and the broad distribution of 1100 channel in TOF axis is neutron distribution. The pulse height region from the 150 to 200 channel is due to the thermal activation events, because the counts of this region decreased as time went on from the beam off. Then, we selected the gamma ray events from the two dimensional plot and obtained the pulse height distributions of prompt gamma rays.

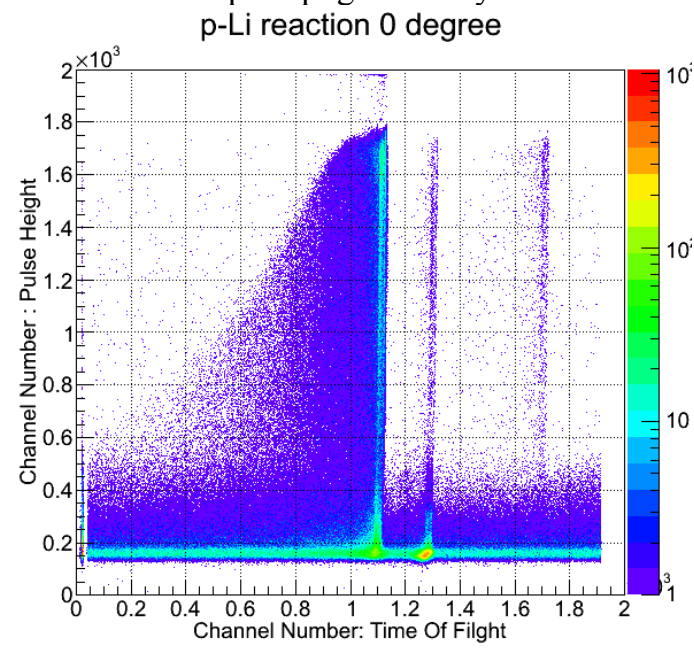

(A)

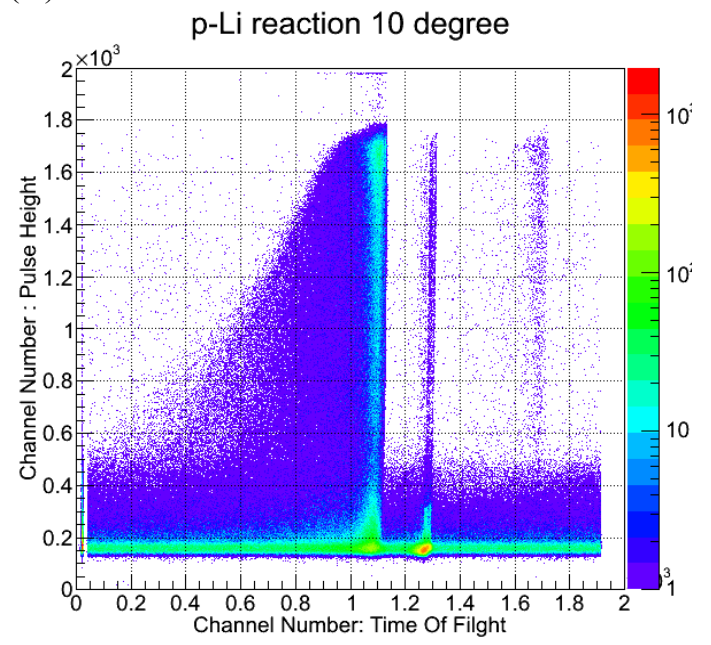

(B)

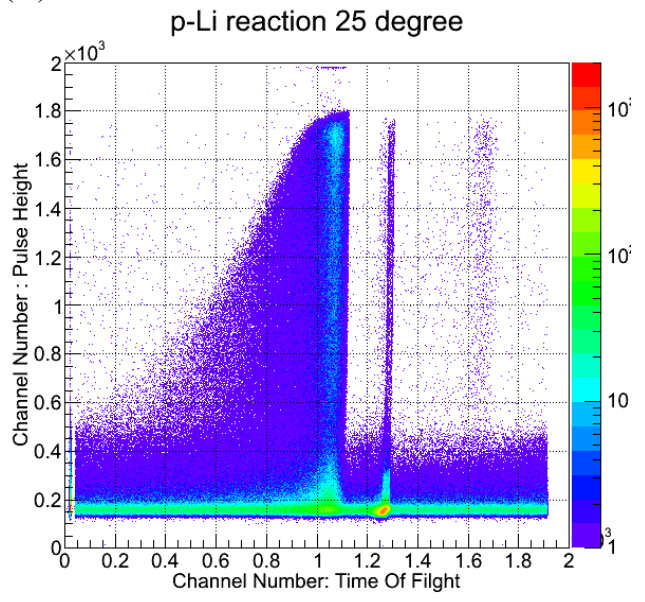

(C)

Figure 1. Two-dimensional plots of pulse height vs. TOF measured for the p-Li neutron beam of (A) 0 deg. (B) $10 \mathrm{deg}$. and (C) $25 \mathrm{deg}$. with respect to the proton beam direction3. 


\section{Results and discussion}

The pulse height spectrum of prompt gamma-ray peak region were obtained by selecting the peak in the two-dimensional distribution plots of pulse height $v s$. time-of-flight. Figures $\mathbf{2}$ and $\mathbf{3}$ show the pulse height spectrum for the each angle of $0,10,25 \mathrm{deg}$. and for the each proton energy of 137 and $200 \mathrm{MeV}$. The Horizontal and vertical axes indicate light output of the $\mathrm{NaI}(\mathrm{Tl})$ scintillator as MeVee (equivalent to $1 \mathrm{MeV}$ electron light output) unit and the number of count per channel per $\mu$-Coulomb, respectively. The spectra were normalized to the integrated charge of the proton beam transported to the beam dump. The red circle and the blue square in Figure 2. indicate the measurements for the 0 degree and for the 25 degree. The red circle, the blue square and the green triangle in Figure 3 indicate the measurements for the 0 degree, the 10 degree and the 25 degree, respectively.

In the both Figures 2 and 3, The events acquired in energy region from 0 to $5 \mathrm{MeV}$ can be ascribed to neutron activation. On the other hand, in the energy region from 5 to $20 \mathrm{MeV}$, the prompt gamma-rays events were observed. The flux of gamma rays due to the $\mathrm{p}$-Li reaction decrease while the angle increases.

In this paper, we assumed the statistical uncertainties only, and the uncertainties are indicated as error bars in Figure 2.

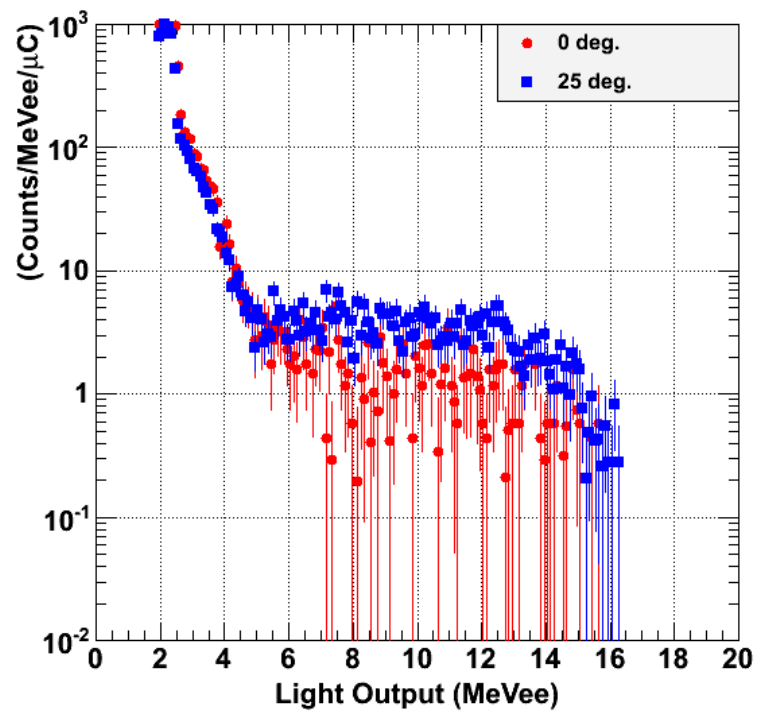

Figure 2. Pulse height spectrum measured in the p-Li of protons of $137 \mathrm{MeV}$.

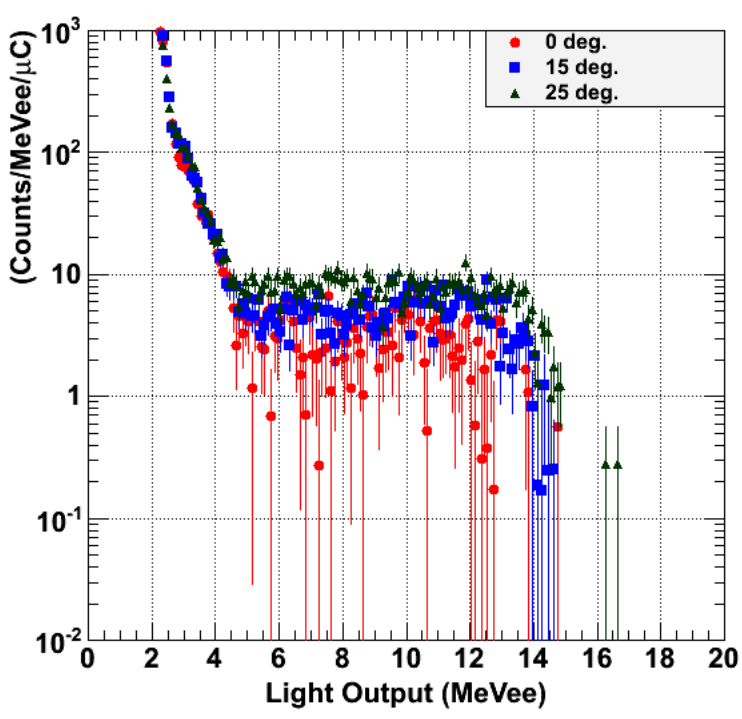

Figure 3. Pulse height spectrum measured in the $\mathrm{p}-\mathrm{Li}$ of protons of $200 \mathrm{MeV}$.

\section{Summary}

We have obtained two-dimensional plot of pulse-height using the $\mathrm{NaI}(\mathrm{Tl})$ scintillator. From the TOF measurement in the $\mathrm{p}-\mathrm{Li}$, we obtained the prompt gamma-ray event in the neutron fields. We will obtain gamma-ray energy spectrum by unfolding method with the response functions for high-energy photons.

\section{Acknowledgements}

We thank all cyclotron operation staff of RCNP Institute for the stable operation of accelerator.

\section{References}

[1] M. J. Berger, J. S. Coursey M. A. Zuker and J. Cahng, Stopping-Power and Range Tables for Electrons, Protons, and Helium Ions: http://www.nist.gov./pml/data/star/index.cfm, (1998).

[2] Y. Iwamoto, M. Hagiwara, T. Matsumoto, A. Masuda, H. Iwase, H. Yashima, T. Shima, A. Tamii and T. Nakamura, Measurements and Monte Carlo calculations of forward-angle secondary-neutron-production cross-sections for 137 and $200 \mathrm{MeV}$ proton-induced reactions in carbon., Nucl. Instrum. Methods. A690 (2012), pp. 10-16.

[3] K. Omata, Y. Fujita, N. Yoshikawa, M. Sekiguchi and Y. Shida, A Data Acquisition System Based on a Personal Computer, INS-Rep.-884, Institute for Nuclear Study, University of Tokyo, (1991). 